\title{
Targeting a heterologous protein to multiple plant organelles via rationally designed 5' mRNA tags
}

Mathias J Voges ${ }^{1,2,3,4}$, Pamela A Silver ${ }^{1,2,3}$, Jeffrey C Way ${ }^{1,2,3}$ and Matthew D Mattozzi $i^{1,2,3^{*}}$

\begin{abstract}
Background: Plant bioengineers require simple genetic devices for predictable localization of heterologous proteins to multiple subcellular compartments.

Results: We designed novel hybrid signal sequences for multiple-compartment localization and characterize their function when fused to GFP in Nicotiana benthamiana leaf tissue. TriTag-1 and TriTag-2 use alternative splicing to generate differentially localized GFP isoforms, localizing it to the chloroplasts, peroxisomes and cytosol. TriTag-1 shows a bias for targeting the chloroplast envelope while TriTag-2 preferentially targets the peroxisomes. TriTag-3 embeds a conserved peroxisomal targeting signal within a chloroplast transit peptide, directing GFP to the chloroplasts and peroxisomes.

Conclusions: Our novel signal sequences can reduce the number of cloning steps and the amount of genetic material required to target a heterologous protein to multiple locations in plant cells. This work harnesses alternative splicing and signal embedding for engineering plants to express multi-functional proteins from single genetic constructs.
\end{abstract}

Keywords: Protein targeting, Alternative splicing, Signal embedding, Localization, Peroxisome, Chloroplast, Cytosol, Organelle, Nicotiana benthamiana

\section{Background}

Plant cells harbor many distinct compartments that share some overlapping function, or are functionally associated in metabolic pathways and development. To enable complex metabolic engineering, plant engineers will require tools to direct single transgenes to multiple compartments. For example, re-engineering photorespiration $[1,2]$ and isoprenoid synthesis $[3,4]$ will involve both the chloroplasts and peroxisomes.

A number of synthetic N-terminal and C-terminal extensions are readily available to target heterologous proteins to desired subcellular compartments, such as the chloroplast, peroxisome, mitochondrion, endoplasmic reticulum or the nucleus. Issues around protein targeting have arisen in (1) studying protein function in a coordinated fashion [5,6], (2) improving holistic plant metabolic

\footnotetext{
*Correspondence: matt.mattozzi@wyss.harvard.edu

'Wyss Institute for Biologically Inspired Engineering, Harvard University, Boston, MA 02115, USA

${ }^{2}$ Department of Systems Biology, Harvard Medical School, Boston, MA 02115, USA Full list of author information is available at the end of the article
}

engineering efforts [7-9] and (3) increasing yields attained by molecular farming and other protein factory applications [10]. One approach to target proteins to more than one location involves cloning multiple genetic copies, each containing a different localization peptide. Each copy must be introduced by successive retransformation, or alternatively, by backcrossing single transforms [11]. These procedures are time-intensive and yield transformants with multiple spatially distinct copies of a protein expression cassette. Coordinate expression may not be ensured due to context-dependent regulatory effects and/or homologybased silencing [12]. Although dual targeting to certain organelles may instead be achieved by adding a second localization peptide [10], this approach is limited to the possible combinations that can be made from available $\mathrm{N}$ and C-terminal extensions.

Here we describe a simple technique for targeting of transgenic proteins to multiple organelles, specifically the chloroplast, peroxisome, and cytosol. This combination of organelles is particularly interesting due to their close functional association in photorespiration, 
isoprenoid biosynthesis, $\beta$-oxidation and other metabolic processes $[3,13,14]$.

\section{Results}

Design for multiple-compartment localization by alternative splicing: TriTag- 1 and TriTag- 2

To construct TriTag-1 and TriTag-2, a chloroplasttargeting region $(\mathrm{CTPa})$ was taken from protein-Lisoaspartate methyltransferase (PIMT2, At5g50240). PIMT2 is a ubiquitous repair protein, converting exposed isoaspartate residues to aspartate or asparagine residues in aging polypeptides $[15,16]$. Various mRNAs produced from PIMT2 are produced by alternative transcription initiation sites and alternative splicing events [16]. In nature, different isoforms are often produced from an individual gene, via the exclusion or inclusion of coding sequences from its mRNA by alternative splicing [17-19]. The spliceforms produced from the 3 ' transcription initiation site target the protein to the chloroplast when the targeting sequence is retained, and to the cytosol when it is not.

A peroxisome targeting sequence, PTS2, containing the $\mathrm{RLx}_{5} \mathrm{HL}$ nonapeptide [20], was taken from the transthyretin-like $\mathrm{S}$-allantoin synthase gene (TTL; At5g58220). This synthase catalyzes two steps in the allantoin biosynthesis pathway [21]. At least two spliceforms are produced from TTL from internal alternative acceptor junctions. The translated proteins are targeted to the peroxisome if they retain the internal PTS2 site and to the cytosol if the site is removed [21].

Harnessing the sequences attained from the above genes, we designed two novel 5' mRNA tags (TriTag-1 and TriTag-2) that targeted the translated GFP to chloroplast, peroxisome and/or cytosol using alternative splicing (Figure 1). An initial pre-mRNA comprising of the entire gene is initially transcribed. This pre-mRNA is then alternatively spliced. The terms "donor" and "acceptor" sites refer to the $5^{\prime}$ (GT) and 3' (AG) splicing

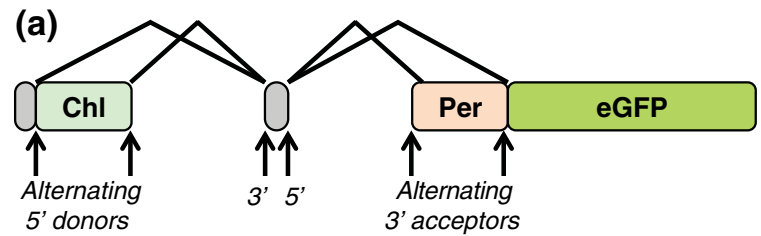

(c)

ATGGAGgtatgttctcttgccaggaatctctgcttcagtttattctcaacacataaggtatac \begin{tabular}{ll|llllllllllllllllll} 
M & E & V & C & S & L & A & R & N & L & C & F & S & L & F & S & T & H & K
\end{tabular}

aaatgggttatttggtgtttctctgtgttgtgtgactgattttgtgcttatagacgattttta atatgttgatggtgttagcaattccagAGTGGAACTGGCTCGAGCGGCGACAGCTCTAGCTCT $\begin{array}{lllllllllllll}S & G & T & G & S & S & G & D & S & S & S & S\end{array}$

CCTGTTTCAACAAAACCTCAAggtatattgatgatttaccaaatcttttccttgtcaaagttt $\begin{array}{lllllll}P & V & S & T & K & P & Q\end{array}$

tgtgtttgactgtgtgggtttgaacctgttaggattcagtatgatatcaagtatgtgtctttt ggaatacaaggatttacccttatggctatctttgttatctgtgtgacctttctactttctcg

ctttgtaagatcgtctgagaatcattggagggcatttgaatgttgcagCTGAAGCAATG $\begin{array}{llllllllllllllllllllll}D & R & L & R & I & I & G & G & H & \text { L } & \text { N } & \text { V } & \text { A } & \text { A } & \text { E } & \text { A } & \text { M }\end{array}$

(e)

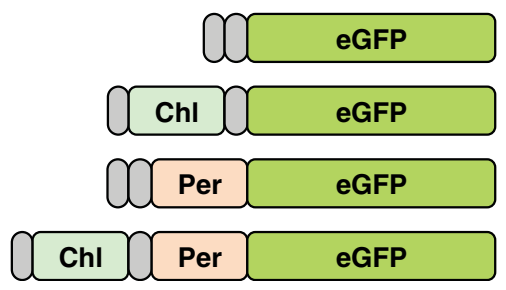

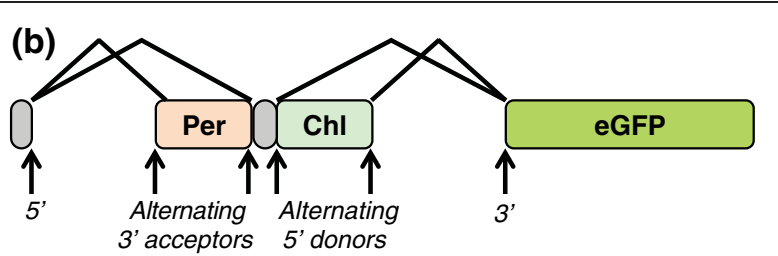

(d)

ATGGACAGCTCTAGCTCTCCTGTTTCAACAAAACCTCAAGgtatattgatgatttaccaaatc $\begin{array}{lllllllllllll}M & D & S & S & S & S & P & V & S & T & K & P & Q\end{array}$

ttttccttgtcaaagttttgtgtttgactgtgtgggtttgaacctgttaggattcagtatgat atcaagtatgtgtcttttggaatacaaggatttaccettatggctatctttgttatctgtgtg accttttctactttctcgctttgtaagatcgtctgagaatcattggagggcatttgaatgtto $\begin{array}{lllllllllllll}D & R & L & R & I & I & G & G & H & L & N & \text { V }\end{array}$ cagCTGAAGCAATGGAGgtatgttctcttgccaggaatctctgcttcagtttattctcaacac $\begin{array}{llllllllllllllllllllll}A & A & E & A & M & E & \text { V } & \text { C } & S & L & A & R & N & L & C & F & S & L & F & S & T\end{array}$ ataaggtatacaaatgggttatttggtgtttctctgtgttgtgtgactgattttgtgcttata $\mathrm{H}$ K

gacgattttaatatgttgatggtgttagcaattcCagAGTGGAACTGGCTCGAGCGGCATG $\begin{array}{lllllllllllll}S & G & T & G & S & S & G & M\end{array}$

(f)

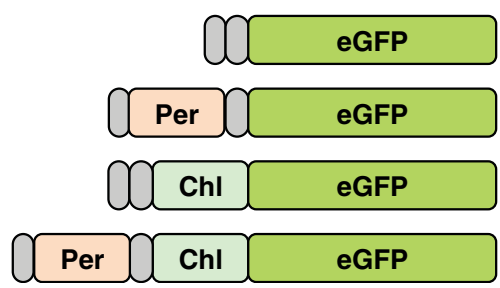

Figure 1 Design of TriTag-1 and TriTag-2, with alternatively spliced chloroplast-targeting PIMT2 and peroxisome-targeting TTL tags. (a, b) Splice diagrams of TriTag-1 (a) and TriTag-2 (b), showing non-targeting sequences (gray), chloroplast targeting sequences (light green), peroxisome targeting sequences (tan), and the enhanced GFP coding sequence used in transient expression experiments. (c, d) Design of TriTag-1 (c) and TriTag-2 (d) sequences. The ATG codon at the end corresponds to the first residue of the GFP open reading frame. Alternatively spliced targeting regions are highlighted. Donor and acceptor dimers are underlined. The light green DNA sequences derive from the PIMT2 $5^{\prime}$ coding region [16] and include sequences required for chloroplast targeting (green). The light tan DNA sequences derive from the TTL $5^{\prime}$ coding region [21] and include sequences encoding the peroxisome targeting sequence (tan). (e, f) Final mRNAs species resulting from alternative splicing of TriTag-1 (e) and TriTag-2 (f). 
junctions, respectively. For example, in Figure 1a, two 5' donor junctions flank an mRNA sequence that encodes a chloroplast-targeting tag. The resultant protein may or may not exhibit a chloroplast-targeting tag, depending on whether the encoding pre-mRNA was excised as an intron.

TriTag-1 contains the elements in this order: a short sequence of PIMT2 containing the start codon, two alternative donor sites flanking chloroplast transit peptide CTPa, a single acceptor site, a short exon that encodes glycine and serine residues, a single donor site, and two alternative acceptor sites flanking the peroxisome targeting sequence PTS2 of the TTL gene (Figure 1a,c). In TriTag- 2 the positions of the sequences taken from genes PIMT2 and TTL are reversed (Figure 1b,d). Both tags are designed so that the two alternative splicing events occur independently of each other. As a result, mRNAs encoding chloroplast, peroxisomal, and cytoplasmically localized proteins are expected (Figure 1e,f).

\section{Design for dual-targeting by signal embedding: TriTag-3}

For targeting to two intracellular locations with a single $\mathrm{N}$-terminal extension, we embedded a peroxisome targeting sequence within a chloroplast targeting sequence (TriTag-3, Figure 2b,d). The 9-aa TTL peroxisometargeting peptide PTS2 $[15,16]$ was placed within the chloroplast targeting region from the ribulose-1,5biphosphate carboxylase (RuBisCO) small-subunit $r b c S 1$ (CTPb, Figure 2a,c, GenBank: X69759.1) [22], substituting for a poorly conserved segment in the CTP that is predicted to form an unfolded segment (determined by the PROFbval tool on the ROSTLAB server [23]). Specifically, the amino acids closest to the $\mathrm{N}$-terminus of the protein are the most effective at differentiating between targeting to the chloroplast and the mitochondria. Inspection of the A. thaliana chloroplast-targeted proteins revealed a decrease in conservation of CTPs toward the C-terminus $[24,25]$. Based on these findings, we placed PTS2 at the 40th amino acid. The resulting targeting peptide, TriTag-3, retains a predicted structure similar to the native $\mathrm{CTPb}$ in terms of flexibility. We determined that proteins containing the N-terminal TriTag-3 extension would be targeted to the peroxisomes and chloroplasts using TargetP [26] and PeroxisomeDB 2.0 [27].

\section{Subcellular localization of GFP controls in transient assays} We tested the targeting properties of the TriTag-GFP fusions in Nicotiana benthamiana leaf epidermal cells using biolistic particle delivery (Bio-Rad Helios Gene Gun) for transient expression. This method allows for rapid transient expression of GFP in a few scattered cells per leaf. This is ideal for observing GFP expression in vivo via fluorescence in a single cell in isolation [17,28]. Expression was controlled by the $\mathrm{P}_{\text {ENTCUP2 }}$ constitutive promoter and the nopaline synthetase (NOS) termination signal [29]. Images were taken by confocal microscopy (Leica SP5 X MP, Buffalo Grove, IL 60089 United States) 48-96 hours after particle delivery. The subcellular fluorescent localization patterns in transfected leaf tissue were compared to chlorophyll autofluorescence; untagged GFP, and constructs designed for expression in the chloroplasts and peroxisomes as controls. A diagram summarizing a typical tobacco leaf epidermal cell and observed expression patterns is provided (Figure 3).

Untagged GFP expressed in the cell periphery and a single large organelle we presumed to be the nucleus. (a)

Chl eGFP

(c)

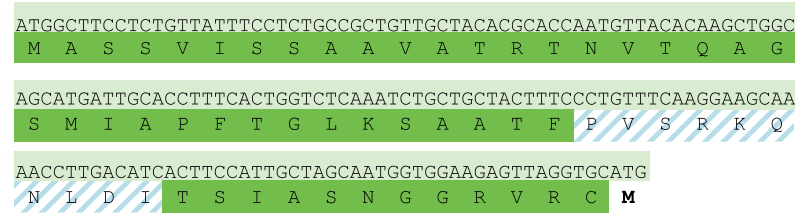

(b)

\begin{tabular}{|l|l|l|}
\hline Chl & Per & eGFP \\
\hline
\end{tabular}

(d)

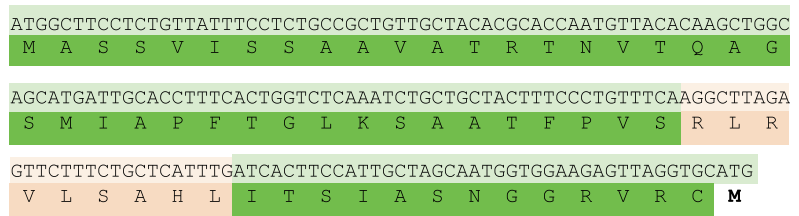

Figure 2 Design of TriTag-3, containing peroxisome-targeting $T L L$ tag embedded within the chloroplast-targeting rbcS1 tag.

(a, b) Diagrams of potato Rubisco rbcS1 chloroplast targeting tag CTPb (a) and TriTag-3 (b), showing chloroplast targeting sequences (light green), peroxisome targeting sequences (tan), flexible regions (diagonals), and the enhanced GFP coding sequence used in transient expression experiments. (c, d) CTPb (c) and TriTag-3 (d) sequences. The ATG codon at the end corresponds to the first residue of the GFP open reading frame. The light green DNA sequences derive from the rbcS1 5' coding region [1] and encode a chloroplast targeting sequence (green).

The light tan DNA sequences (d) code for a consensus PTS2 signal (tan). The PTS2 sequence is embedded within a flexible region of CTPb (green diagonals). 


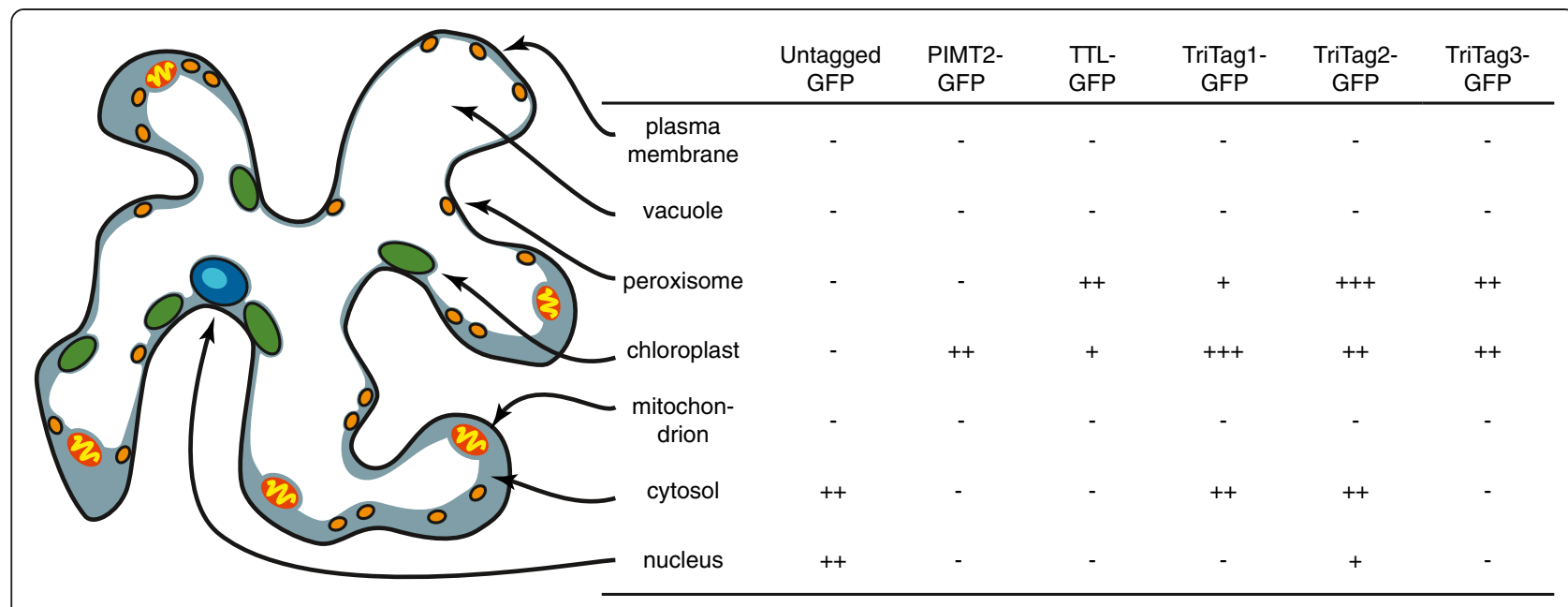

Figure 3 Compartments of a typical tobacco leaf epidermal cell. Here we illustrate their relative sizes and locations within the cell, and the relative expression levels observed via confocal microscopy.

GFP expression in a single organelle per cell that was larger than a chloroplast and not co-localized to a chloroplast was presumed to be in the nucleus. As the vacuole takes up $90 \%$ of the cell volume (Figure 3), expression in the cell periphery is likely cytosolic (Figure 4a,b,c; see also [30]). GFP fused to the chloroplast targeting peptide of PIMT2 [15,16] showed expression in chloroplasts (Figure 4d,e,f). GFP fused to the peroxisome targeting peptide of $T T L[20,21]$ (slightly modified from [21] by the addition of a start codon) showed expression in organelles that resemble peroxisomes (Figure 4g,h,i). The TTLtagged GFP was sometimes localized to a subset of the chloroplasts in the transfected cells, but much weaker than was observed using the TriTag constructs (below).

\section{Subcellular localization of TriTag-1 and TriTag-2 fused GFP}

TriTag- 1 and TriTag-2 were designed to target the resultant protein to either the chloroplast or peroxisome via alternative splicing of the encoding mRNA. The two tags contain the same elements but in complimentary orders (Figure 1). Both tags showed localization to the cytoplasm, chloroplast, and peroxisome (Figures 5 and 6). GFP expression (Figures $5 \mathrm{a}, \mathrm{d}, \mathrm{g}$ and $6 \mathrm{a}, \mathrm{d}, \mathrm{g}$ ) was compared to the autofluorescence of chloroplasts (Figures 5b,e,h and 6b,e,h) and the size and distribution pattern of peroxisomes (Figure 4g,h,i). Transient expression of TriTag-1-GFP resulted in cytosolic and chloroplast localization, with the latter inferred by chlorophyll co-localization in the transfected cell. GFP expression in a single organelle per cell that was larger than a chloroplast and not co-localized to a chloroplast was presumed to be in the nucleus. Additional punctate staining was observed that did not correspond to chloroplasts, but was similar to the expression observed with the TTL (peroxisomal-targeted) vector (Figure $4 \mathrm{~g}, \mathrm{~h}, \mathrm{i}$ ) and was attributed to peroxisomal targeting. Typical peroxisomes are labeled with arrows in Figure 4g,i.

Transiently expressed TriTag-2-GFP (Figure 6) display cytosolic localization, as well as a bright punctate pattern indicating a higher level of peroxisomal targeting and a lower signal in the chloroplasts. Overall, TriTag-1 localized GFP preferentially to the chloroplasts, while TriTag-2 localized this protein to the peroxisomes, with similar targeting to the cytoplasm, as evidenced by GFP localization at the cell periphery and presumably the nucleus.

\section{Subcellular localization of TriTag-3 fused to GFP}

TriTag-3 was designed to contain the TTL peroxisomal targeting sequence within the PIMT2 chloroplast sequence (Figure 2). N. benthamiana epidermal leaf cells transiently expressing TriTag-3-GFP display chloroplast localization and punctate peroxisomal localization (Figure 7). Essentially no GFP was observed in the cytosol. This observation indicates that the hybrid chloroplast/peroxisome targeting sequence is efficiently recognized by the corresponding localization systems, and also that the cytoplasmic plus nuclear localization observed with TriTags 1 and 2 is likely due to mRNAs spliced so that they lack both the peroxisomal and chloroplast targeting sequences.

\section{Discussion}

In this study, we describe simple strategies for localizing a single transgenic protein to multiple cellular compartments in plants. Variation in N-terminal targeting sequences was presumably encoded by alternative splicing of the encoding mRNA or simultaneous function of multiple-targeting sequences as expressed in a single protein. This greatly economizes the amount of DNA transfected. In addition, dual targeting was achieved by 


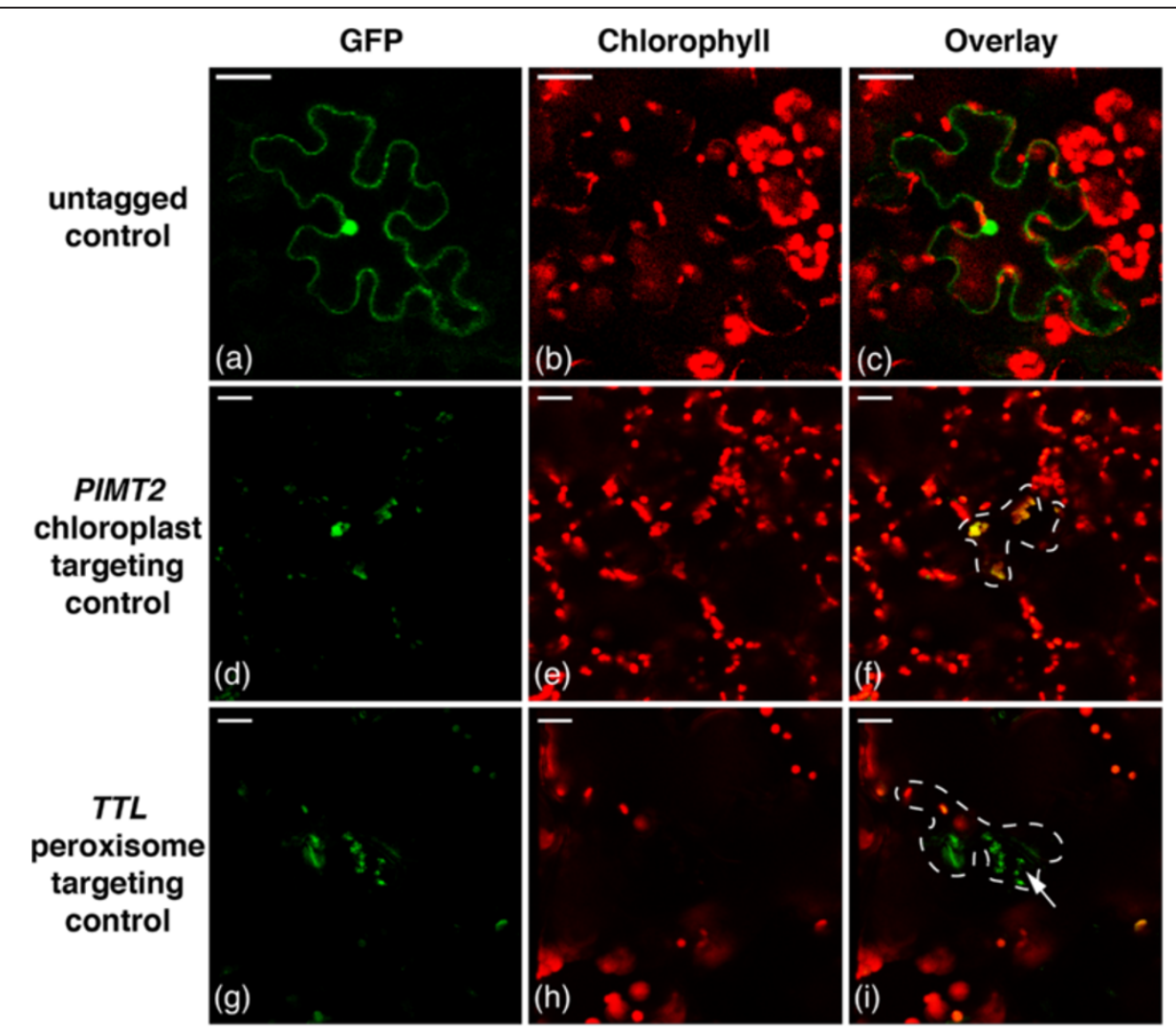

Figure 4 Typical transient expression pattern controls in $\boldsymbol{N}$. benthamiana leaf epidermal cells. GFP channel (a, d, g), chlorophyll autofluorescence $(\mathbf{b}, \mathbf{e}, \mathbf{h})$ and composite $(\mathbf{c}, \mathbf{f}, \mathbf{i})$ images were generated using confocal microscopy. Comparison with a,b and $\mathbf{c}$ allow for identification of non-targeted GFP by a pattern of cytosolic, plasma membrane and nuclear expression. The PIMT2-GFP fusion in d, e and f shows expression in the chloroplast. The TTL-GFP fusion in $\mathbf{g}, \mathbf{h}$, and $\mathbf{i}$ shows expression throughout the cell but some preference for small organelles we believe to be peroxisomes (arrow). Scale bar is $25 \mu \mathrm{m}$.

an ambiguous $\mathrm{N}$-terminal signal with elements of chloroplast and peroxisomal targeting sequences. We designed three different short, $\mathrm{N}$-terminal elements for coordinate chloroplast, peroxisome and cytosol targeting, termed 'TriTags". TriTag-1 and TriTag-2 (Figure 1) were designed by combining naturally occurring DNAs encoding alternatively spliced mRNAs that direct the encoded proteins to either the chloroplast plus cytoplasm [16] or the peroxisome plus cytoplasm [21]. TriTag-3 (Figure 2) does not rely on alternative splicing and consists of a chloroplast targeting sequence in which a naturally unstructured portion has been replaced with a peroxisomal targeting sequence [31].

The TriTags function in vivo to target GFP in Nicotiana benthamiana leaf epidermal cells (summarized in Figure 3). We compared confocal images of the TriTags to controls of untagged GFP, and GFP with N-terminal tags that had previously been shown to target chloroplasts and peroxisomes [15,16,20,21]. Plasmid DNA was delivered into leaf cells by standard biolistic transfection. Untagged GFP was localized to the cytoplasm and nucleus, with some nuclear localization being expected because the nuclear pore has a large, aqueous channel that permits entry of molecules up to about $70 \mathrm{kD}$. TriTag- 1 and TriTag- 2 mediated GFP expression in the chloroplast, peroxisome, and cytoplasm (plus nucleus), with TriTag-1 showing a slight preference for the chloroplast over peroxisome and TriTag-2 showing the opposite behavior. TriTag-3 mediated strong localization to both the peroxisome and chloroplast, but not detectably to the cytoplasm. These behaviors suggest that alternatively spliced forms of TriTag- 1 and TriTag- 2 are being produced (Figures 1 and 3 ).

The re-engineering of photorespiration pathways [1] illustrates the potential utility of such multiple-targeting elements. Normally during photorespiration, glycolate is generated in the chloroplast and then transported into the cytoplasm and then into the peroxisome, where it is oxidized to glyoxylate in an $\mathrm{O}_{2}$-dependent reaction. The reduction of oxygen, rather than $\mathrm{NAD}(\mathrm{P})^{+}$as an oxidizing agent represents a waste of reducing equivalents and energy. Kebeish et al. [1] engineered plants to express in the chloroplast and $\mathrm{NAD}^{+}$-dependent bacterial glycolate metabolizing pathway and found this enhanced the 


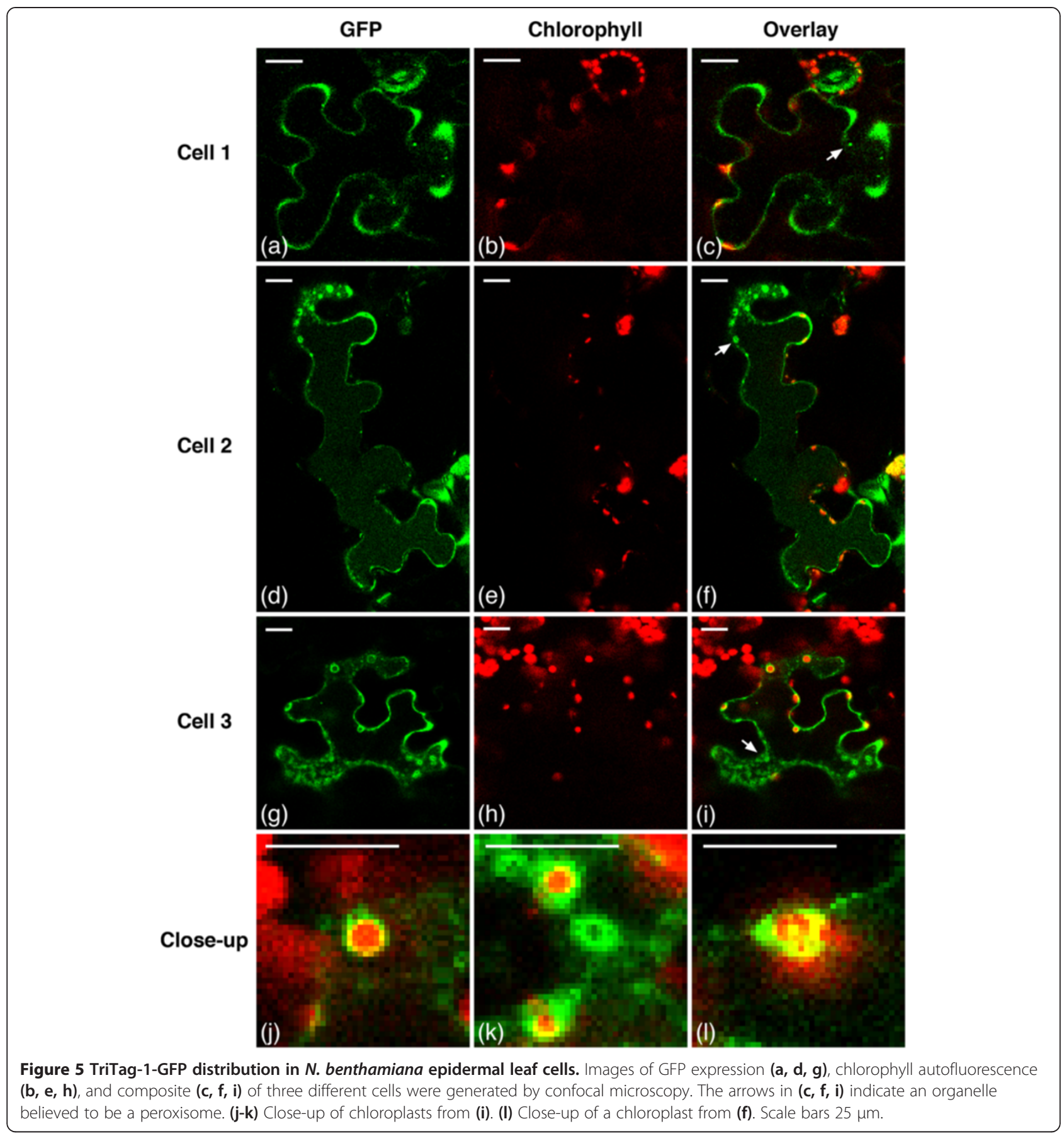

growth of light-limited Arabidopsis. In this situation, the added bacterial pathway competes with transport of glycolate from the chloroplast into the cytoplasm. Expression of the pathway in the cytoplasm and peroxisome could further enhance the amount of glycolate that is metabolized by this more efficient pathway. However, as the pathway involves five polypeptide coding sequences, expression of all proteins in three compartments could be prohibitive.
Our results also suggest that novel alternative splicing systems can be engineered in a straightforward manner. One risk in designing such systems is that it is difficult to predict the efficiency of a novel alternatively spliced system. Future designs may use different alternativelyspliced base genes, whose splice ratios may be better known for quantitation. Further experiments with a more stable vector delivery system (e.g. Agrobacterium 


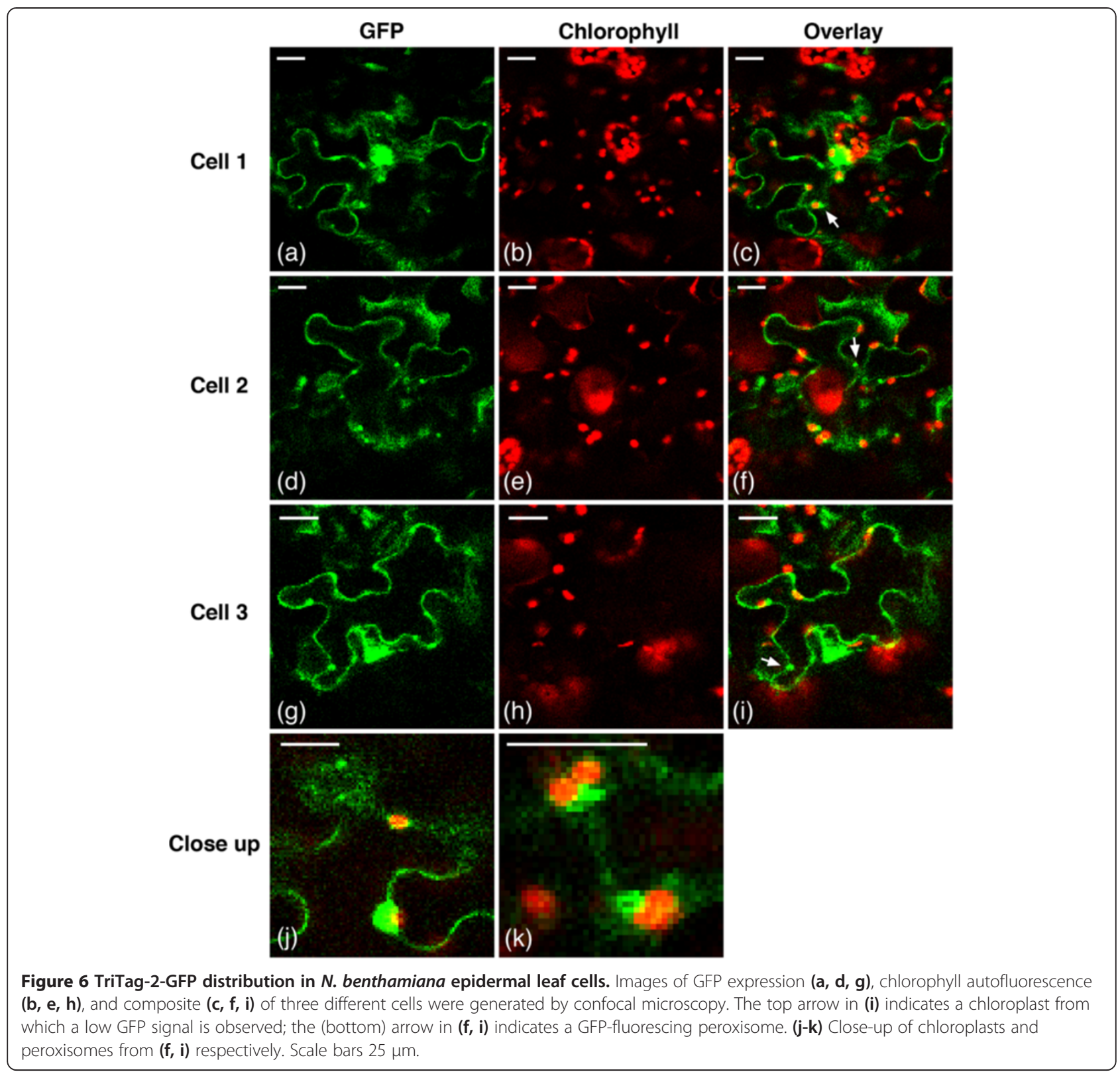

transfection) could give us additional material for quantitative PCR or changes in expression over time, and further inform future designs based on engineered alternative splicing.

\section{Conclusions}

Plant metabolic engineering remains a formidable effort in terms of time and resources. The field requires simple and efficient technologies for transforming plants with multi-functional proteins. Our system with engineered alternative splicing could be used to target a single transgene to multiple locations, namely the chloroplast, cytosol, and peroxisome. In addition, we demonstrated that a peroxisomal signal embedded within a chloroplast signal allows dual targeting of the transgene. These devices may reduce time and resources spent on plant metabolic engineering.

\section{Methods}

\section{Strains and plasmids}

E. coli K12 strains (NEB Turbo, New England Biolabs) were used as plasmid hosts for cloning work on binary vectors for transient expression and/or stable genomic integration. Plasmids (Table 1), were constructed with traditional cloning methods [32], BglBricks [33], BioBricks [34], or Gibson assembly [35]. E. coli K12 cells were grown in Luria-Bertani medium with appropriate antibiotics $(100 \mu \mathrm{g} / \mathrm{mL}$ kanamycin). 


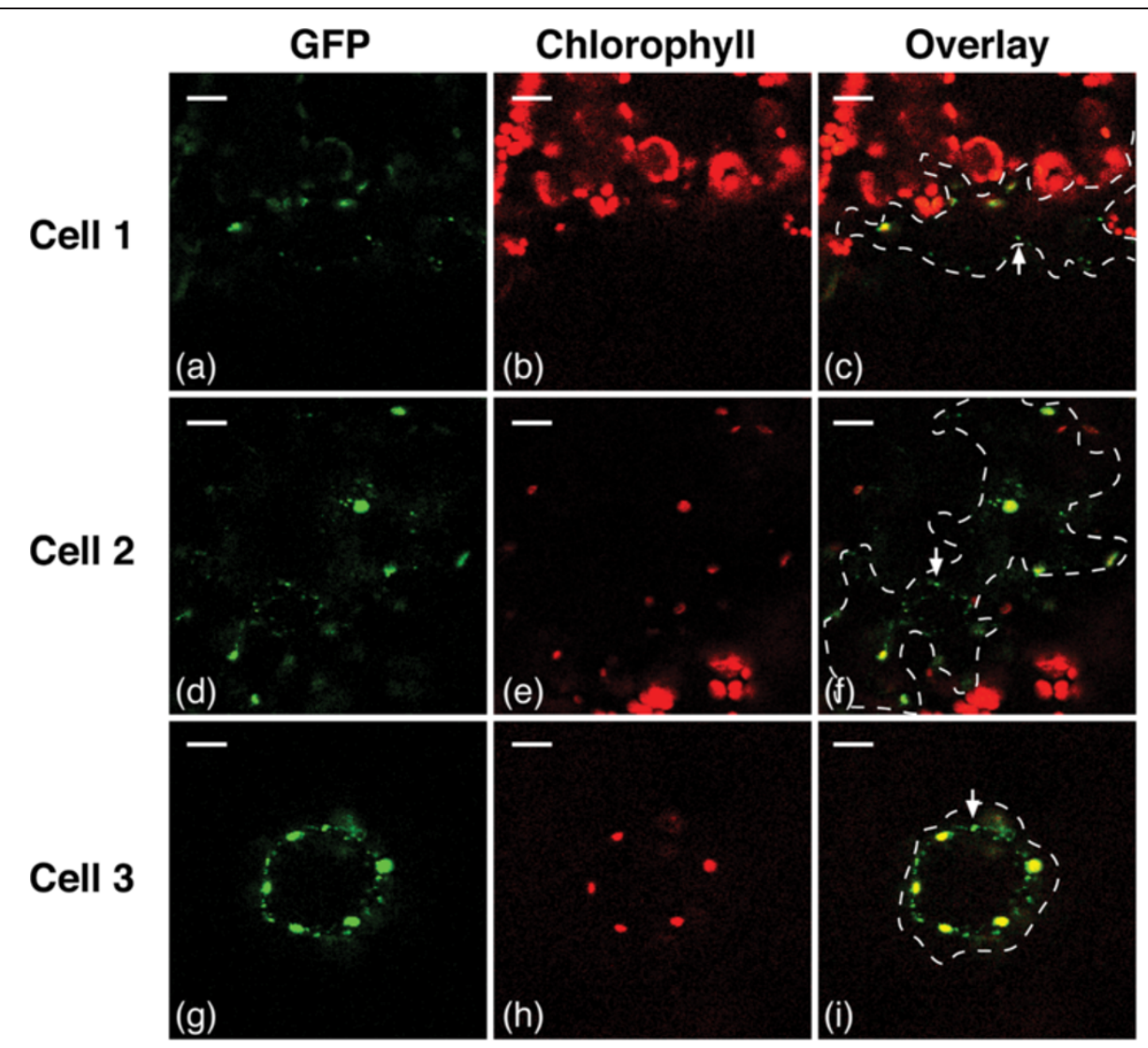

Figure 7 TriTag-3-GFP distribution in $\mathbf{N}$. benthamiana leaf epidermal cells. Images of GFP expression $(\mathbf{a}, \mathbf{d}, \mathbf{g})$, chlorophyll autofluorescence $(\mathbf{b}, \mathbf{e}, \mathbf{h})$, and composite $(\mathbf{c}, \mathbf{f}, \mathbf{i})$ of three different cells were generated by confocal microscopy. Arrows indicate fluorescing peroxisomes. While a clear cellular periphery is not distinguishable in the GFP signal, essentially no fluorescence is observed in the cytosol. Scale bars $25 \mu \mathrm{m}$.

\section{TriTag synthesis and cloning}

TriTag-1, TriTag-2 and TriTag-3 were synthesized (GeneBlocks, IDT, Coralville, IA), and cloned in-frame $5^{\prime}$ of the soluble modified GFP (smGFP) using Gibson assembly [35]. This modified GFP contains three site-

\section{Table 1 Plasmids constructed in this study}

PORE-GFP

PORE-PIMT2-GFP

PORE-TTL-GFP

pORE-TriTag-1-GFP

pORE-TriTag-2-GFP

pORE-TriTag-3-GFP
PORE binary vector expressing untagged soluble modified GFP (smGFP) controlled by the PENTCUP2 promoter [29].

PORE vector expressing $A$. thaliana codonoptimized GFP, with an N-terminal chloroplast targeting peptide of protein-L-isoaspartate methyltransferase (PIMT2) [15,16].

pORE vector expressing $A$. thaliana codon-optimized GFP, with an N-terminal start codon and peroxisome-targeting peptide of transthyretin-like S-allantoin synthase (TTL) [20,21]

pORE vector expressing TriTag-1-fused GFP controlled by the pENTCUP2 promoter.

pORE vector expressing TriTag-2-fused GFP controlled by the PENTCUP2 promoter.

PORE vector expressing TriTag-3-fused GFP controlled by the pENTCUP2 promoter. directed mutations that increase the protein's solubility and fluorescence intensity [36]. Based on splice site prediction with NetPlantGene [37], we predicted that the processed spliceforms of TriTag- 1 and TriTag 2 encodes for GFP variants containing regions for chloroplast targeting, peroxisomal targeting or neither. Spliceforms other than those found using NetPlantGene would either incorporate a stop codon or lack organelle-targeting information, causing premature translation or sole targeting to the cytosol, respectively.

\section{Plant material}

All plants were incubated at $16-20^{\circ} \mathrm{C}$ in a $16 / 8$ hour light/ dark cycle and watered twice weekly. Peat-based soilfree media (Metromix, SunGro Horticulture, Vancouver, Canada) was autoclaved $45 \mathrm{~min}$ before use. Leaves from 3-5 month old Nicotiana benthamiana seedlings plants were collected for bombardment.

\section{Biolistic delivery}

DNA-gold particle complexes for biolistic delivery were prepared according to manufacturer's instructions for use with the Helios Gene Gun (Bio-Rad, Hercules, CA) 
as follows: Plasmid DNA $(50 \mu \mathrm{g})$ containing the tagged GFP gene was pelleted onto $1 \mu \mathrm{m}$ gold particles $(6-8 \mathrm{mg})$ in a spermidine $(100 \mu \mathrm{L}, 0.05 \mathrm{M})$ and $\mathrm{CaCl}_{2}(100 \mu \mathrm{L}$, $1.0 \mathrm{M})$ mixture and resuspended in a polyvinylpyrrolidone/EtOH solution $(5.7 \mathrm{mg} / \mathrm{mL})$. The resulting suspension was deposited onto the inside surface area of Tygon plastic tubing (o.d. $=2 \mathrm{~mm}$ ) and diced into cartridges facilitated by the Tubing Prep Station (Bio-Rad, Hercules, CA). Cartridges were stable up to 6 months dessicated at $4^{\circ} \mathrm{C}$. The underside of Nicotiana benthamiana leaves were transformed biolistically using the Helios Gene Gun (BioRad, Hercules, CA) at 150-250 psi He [38]. The leaves were placed on wet filter paper in Petri dishes and stored on a bench-top under ambient lighting and room temperature for 48 hours before imaging analysis.

\section{Target control proteins}

As expected, control proteins showed untagged smGFP was distributed extensively in the cytosol and nucleus (Figure $4 \mathrm{a}, \mathrm{b}, \mathrm{c}$ ), but not the vacuole, which makes up the bulk of the plant cell volume. This localization pattern matches previous untagged GFP localization studies [30]. Cytosolic and chloroplast localization controls were determined by transient expression of GFP fused to the PIMT2 chloroplast targeting peptide and the native autofluorescence of chlorophyll (Figure 4d,e,f). Peroxisomal localizations were determined by comparing images to transient expression of GFP fused to the TTL peroxisomal targeting peptide, which matches previous localization studies [21].

\section{Prediction software}

Splice junctions within the TriTag-1 and TriTag-2 sequences were predicted using the NetPlantGene server [37]. Targeting to the chloroplast and peroxisome of the TriTag- 1 and TriTag- 2 splice variants and TriTag- 3 were predicted using TargetP [26] and PeroxisomeDB 2.0 [27]. Peptide structures of $\mathrm{CTPb}$ and TriTag-3 were determined using PROFbval on the ROSTLAB server [23].

\section{Imaging and processing}

Bombarded leaves were diced and placed on glass slides in $0.1 \%$ Triton-X100 and imaged by fluorescence confocal microscopy (excitation at $489 \mathrm{~nm}$, detection at 500-569 for GFP and 630-700 for chlorophyll autoflourescence) using a 40x water-based objective (numerical aperture 1.10).

\footnotetext{
Abbreviations

PTS2: Peroxisome targeting signal 2; TTL: Arabidopsis transthyretin-like S-allantoin synthase gene; CTP: Chloroplast targeting peptide; PIMT2: Arabidopsis protein-L-isoaspartate methyltransferase gene; CTPa: Chloroplast targeting peptide from PIMT2; rbcS1: Solanum tuberosum ribulose-1,5-biphosphate carboxylase (RuBisCO) small-subunit gene; CTPb: Chloroplast targeting peptide from rbcS1; smGFP: Soluble modified green fluorescent protein.
}

Competing interests

PAS, JCW, and MdM have founded in Chimerion Biotechnology, Inc., on related research.

\section{Authors' contributions}

PAS, JCW, and MdM conceived of the research; MV and MdM designed the study and performed the experiments; MV, JCW and MdM analyzed the data and wrote the manuscript. All authors read and approved the final manuscript.

\section{Acknowledgments}

The authors would like to thank Jen Sheen of Massachusetts General Hospital for the generous donation of Nicotiana benthamiana seedlings. Jen Bush helped us greatly in advice in growing plants and setting up a growth chamber area. Tom Ferrante, Garry Cuneo, and Danny Selgrade of the Wyss Institute offered crucial help with confocal microscopy. We would especially like to thank Don Ingber of Children's Hospital Boston and the Wyss Institute for the generous donation of the BioRad Helios Gene Gun and associated supplies. Funding for this project was provided through a cooperative agreement with the Department of Energy Advanced Research Projects Agency (ARPA-E Award \# DE-000079) for PAS, JCW, and MdM, and through Chimerion Biotechnologies, Inc. for MJV.

\section{Author details}

${ }^{1}$ Wyss Institute for Biologically Inspired Engineering, Harvard University, Boston, MA 02115, USA. ${ }^{2}$ Department of Systems Biology, Harvard Medical School, Boston, MA 02115, USA. ${ }^{3}$ Chimerion Biotechnology Inc, Cambridge, MA 02138, USA. ${ }^{4}$ Department of Biotechnology, Technische Universiteit Delft, 2628 BC, Delft, Netherlands.

Received: 6 March 2013 Accepted: 17 August 2013

Published: 8 September 2013

\section{References}

1. Kebeish R, Niessen M, Thiruveedhi K, Bari R, Hirsch H-JJ, Rosenkranz R, Stäbler N, Schönfeld B, Kreuzaler F, Peterhänsel C: Chloroplastic photorespiratory bypass increases photosynthesis and biomass production in Arabidopsis thaliana. Nat Biotechnol 2007, 25:593-599.

2. Maier A, Fahnenstich H, von-Caemmerer S, Engqvist MKM, Weber APM, Flügge U-I, Maurino VG: Transgenic introduction of a glycolate oxidative cycle into A. thaliana chloroplasts leads to growth improvement. Front Plant Sci 2012, 3(February):38.

3. Sapir-Mir M, Mett A, Belausov E, Tal-Meshulam S, Frydman A, Gidoni D, Eyal Y: Peroxisomal localization of Arabidopsis isopentenyl diphosphate isomerases suggests that part of the plant isoprenoid mevalonic acid pathway is compartmentalized to peroxisomes. Plant Physiol 2008, 148:1219-1228

4. Kumar S, Hahn FM, Baidoo E, Kahlon TS, Wood DF, McMahan CM, Cornish K, Keasling JD, Daniell H, Whalen MC: Remodeling the isoprenoid pathway in tobacco by expressing the cytoplasmic mevalonate pathway in chloroplasts. Metab Eng 2012, 14:19-28.

5. Hooks KB, Turner JE, Graham IA, Runions J, Hooks MA: GFP-tagging of Arabidopsis acyl-activating enzymes raises the issue of peroxisomechloroplast import competition versus dual localization. J Plant Physiol 2012, 169:1631-1638.

6. Zhang $X, \mathrm{Hu}$ J: The Arabidopsis chloroplast division protein DYNAMINRELATED PROTEIN5B also mediates peroxisome division. Plant Cell 2010, 22:431-442

7. Baudisch B, Klösgen RB: Dual targeting of a processing peptidase into both endosymbiotic organelles mediated by a transport signal of unusual architecture. Mol Plant 2012, 5:494-503.

8. Brandão MM, Silva-Filho MC: Evolutionary history of Arabidopsis thaliana aminoacyl-tRNA synthetase dual-targeted proteins. Mol Biol Evol 2011, 28:79-85.

9. Severing El, van-Dijk AD, van-Ham RC: Assessing the contribution of alternative splicing to proteome diversity in Arabidopsis thaliana using proteomics data. BMC Plant Biol 2011, 11:82

10. Hyunjong B, Lee D-S, Hwang I: Dual targeting of xylanase to chloroplasts and peroxisomes as a means to increase protein accumulation in plant cells. J Exp Bot 2006, 57:161-169. 
11. Que Q, Chilton M-DM, de-Fontes CM, He C, Nuccio M, Zhu T, Wu Y, Chen JS, Shi L: Trait stacking in transgenic crops: challenges and opportunities. GM Crops 2010, 1:220-229.

12. Dafny-Yelin M, Tzfira T: Delivery of multiple transgenes to plant cells. Plant Physiol 2007, 145:1118-1128.

13. Baker A, Graham IA, Holdsworth M, Smith SM, Theodoulou FL: Chewing the fat: beta-oxidation in signalling and development. Trends Plant Sci 2006, 11:124-132.

14. Peterhansel C, Horst I, Niessen M, Blume C, Kebeish R, Kürkcüoglu S, Kreuzaler F: Photorespiration. The Arabidopsis Book 2010, 8:e0130.

15. Lowenson JD, Clarke S: Recognition of D-aspartyl residues in polypeptides by the erythrocyte L-isoaspartyl/D-aspartyl protein methyltransferase. Implications for the repair hypothesis. J Biol Chem 1992, 267:5985-5995.

16. Dinkins RD, Majee SM, Nayak NR, Martin D, Xu Q, Belcastro MP, Houtz RL, Beach CM, Downie AB: Changing transcriptional initiation sites and alternative 5 '- and 3'-splice site selection of the first intron deploys Arabidopsis protein isoaspartyl methyltransferase2 variants to different subcellular compartments. Plant J 2008, 55:1-13.

17. Reddy ASN, Rogers MF, Richardson DN, Hamilton M, Ben-Hur A: Deciphering the plant splicing code: experimental and computationa approaches for predicting alternative splicing and splicing regulatory elements. Front Plant Sci 2012, 3(February):18.

18. Black DL: Mechanisms of alternative pre-messenger RNA splicing. Annu Rev Biochem 2003, 72:291-336.

19. Kalsotra A, Cooper TA: Functional consequences of developmentally regulated alternative splicing. Nat Rev Genet 2011, 12:715-729.

20. Lanyon-Hogg T, Warriner SL, Baker A: Getting a camel through the eye of a needle: the import of folded proteins by peroxisomes. Biol Cell 2010 102:245-263.

21. Reumann S, Babujee L, Ma C, Wienkoop S, Siemsen T, Antonicelli GE, Rasche N, Lüder F, Weckwerth W, Jahn O: Proteome analysis of Arabidopsis leaf peroxisomes reveals novel targeting peptides, metabolic pathways, and defense mechanisms. Plant Cell 2007, 19:3170-3193.

22. Fritz CC, Herget T, Wolter FP, Schell J, Schreier PH: Reduced steady-state levels of rbcS mRNA in plants kept in the dark are due to differential degradation. Proc Natl Acad Sci U S A 1991, 88:4458-4462.

23. Schlessinger A, Yachdav G, Rost B: PROFbval: predict flexible and rigid residues in proteins. Bioinformatics 2006, 22:891-893.

24. Sadler I, Chiang A, Kurihara T, Rothblatt J, Way J, Silver P: A yeast gene important for protein assembly into the endoplasmic reticulum and the nucleus has homology to DnaJ, an Escherichia coli heat shock protein. J Cell Biol 1989, 109(6 Pt 1):2665-2675.

25. Bhushan S, Kuhn C, Berglund A-K, Roth C, Glaser E: The role of the Nterminal domain of chloroplast targeting peptides in organellar protein import and miss-sorting. FEBS Lett 2006, 580:3966-3972.

26. Emanuelsson $\mathrm{O}$, Brunak $\mathrm{S}$, von-Heijne $\mathrm{G}$, Nielsen $\mathrm{H}$ : Locating proteins in the cell using TargetP, SignalP and related tools. Nat Protoc 2007, 2:953-971.

27. Schlüter A, Real-Chicharro A, Gabaldón T, Sánchez-Jiménez F, Pujol A: PeroxisomeDB 2.0: an integrative view of the global peroxisomal metabolome. Nucleic Acids Res 2010, 38(Database issue):D800-D805

28. Stauffer E, Westermann A, Wagner G, Wachter A: Polypyrimidine tractbinding protein homologues from Arabidopsis underlie regulatory circuits based on alternative splicing and downstream control. Plant $J$ 2010, 64:243-255.

29. Coutu C, Brandle J, Brown D, Brown K, Miki B, Simmonds J, Hegedus DD: PORE: a modular binary vector series suited for both monocot and dicot plant transformation. Transgenic Res 2007, 16:771-781.

30. Li F, Liu W, Tang J, Chen J, Tong H, Hu B, Li C, Fang J, Chen M, Chu C: Rice DENSE AND ERECT PANICLE 2 is essential for determining panicle outgrowth and elongation. Cell Res 2010, 20:838-849.

31. Silva-Filho MC: One ticket for multiple destinations: dual targeting of proteins to distinct subcellular locations. Curr Opin Plant Biol 2003, 6:589-595.

32. Sambrook J, Russell DW: Molecular Cloning: A Laboratory Manual. 3rd edition. Cold Spring Harbor, New York: Cold Spring Harbor Laboratory Press; 2001.

33. Anderson JC, Dueber JE, Leguia M, Wu GC, Goler JA, Arkin AP, Keasling JD: BglBricks: A flexible standard for biological part assembly. J Biol Eng 2010, 4:1.

34. Shetty RP, Endy D, Knight TF: Engineering BioBrick vectors from BioBrick parts. J Biol Eng 2008, 2:5
35. Gibson DG: Enzymatic assembly of overlapping DNA fragments. Methods Enzymol 2011, 498:349-361.

36. Davis SJ, Vierstra RD: Soluble, highly fluorescent variants of green fluorescent protein (GFP) for use in higher plants. Plant Mol Biol 1998, 36:521-528

37. Hebsgaard SM, Korning PG, Tolstrup N, Engelbrecht J, Rouzé P, Brunak S: Splice site prediction in Arabidopsis thaliana pre-mRNA by combining local and global sequence information. Nucleic Acids Res 1996, 24:3439-3452.

38. Woods G, Zito K: Preparation of gene gun bullets and biolistic transfection of neurons in slice culture. J Vis Exp 2008, 12:10-13.

doi:10.1186/1754-1611-7-20

Cite this article as: Voges et al:: Targeting a heterologous protein to multiple plant organelles via rationally designed 5' mRNA tags. Journal of Biological Engineering 2013 7:20.

\section{Submit your next manuscript to BioMed Central and take full advantage of:}

- Convenient online submission

- Thorough peer review

- No space constraints or color figure charges

- Immediate publication on acceptance

- Inclusion in PubMed, CAS, Scopus and Google Scholar

- Research which is freely available for redistribution 